\title{
Performance Analysis of the 2DoF Direct Drive Induction Motor Applying Composite Multilayer Method
}

\author{
Jikai $\mathrm{Si}^{1}$, Lujia Xie ${ }^{1}$, Wenping $\mathrm{Cao}^{2}$, Yihua $\mathrm{Hu}^{3}$, Haichao Feng ${ }^{1}$ \\ ${ }^{1}$ School of Electrical Engineering and Automation, Henan Polytechnic University, Jiaozuo Henan, China \\ ${ }^{2}$ University of Aston, Department of Electrical Engineering, Birmingham, UK \\ ${ }^{3}$ Department of Electrical Engineering and Electronics, University of Liverpool, Liverpool, UK
}

\begin{abstract}
This paper presents a composite multilayer method (CMM) to evaluate the performance of a two-degree-of-freedom (2DoF) direct drive induction motor (2DoFDDIM) whose solid rotor is coated with a copper layer. It includes a rotary part and a linear part. Based on the traditional multilayer theory, a complete 2DoFDDIM CMM computer program importing propagation constants is built. Due to the complex magnetic field in a 2DoFDDIM, this paper mainly analyses it from the perspective of a single DOF motor. An equivalent circuit for the rotary part of the 2DoFDDIM is then derived applying CMM and the two-dimensional magnetic field distribution is obtained by solving Maxwell's equations in motor layers. The developed torques, power factors and stator currents of the rotary part with different slips and the latter two of the linear part at zero speed are calculated by CMM, which are then compared with results from the finite element method (FEM) and experimental results. The computation time of the CMM is far less than that of the FEM. The acceptable accuracy confirms the effectiveness of the CMM for analysis and performance calculations of the 2DoFDDIM.
\end{abstract}

Index Terms - Composite multilayer method, double-layer solid rotor, finite element method, two-degree-of-freedom motor.

\section{Introduction}

Electric motors are one of the components involved that can contribute to energy savings [1]. Two-degree-of-freedom (2DoF) motors or actuators have high mechanical integration and reliability which can eliminate mechanical transmission in the middle of a driver. They can produce rotary, linear and helical motion by themselves, as shown in Fig.1, and are widely utilized in industrial machinery such as boring machines and grinders [2]- [3]. The conventionally complicated structure comprising of a linear motor and a rotary motor can be substituted by one 2Dof motor, which greatly simplifies the system. A few different 
topologies of $2 \mathrm{DoF}$ motors have been proposed and investigated by researchers [2]- [10]. For example, a 2DoF outer rotor spherical actuator [7] and a novel magnetization pattern [8] have been presented for 2DoF rotary-linear actuators. In this paper, a two-degree-of-freedom direct drive induction motor (2DoFDDIM) with an integrated structure is proposed, as shown in Fig. 2.

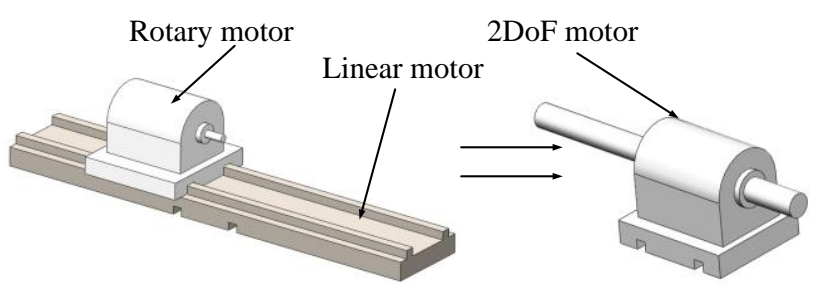

Fig. 1. System simplification by 2DoF motors

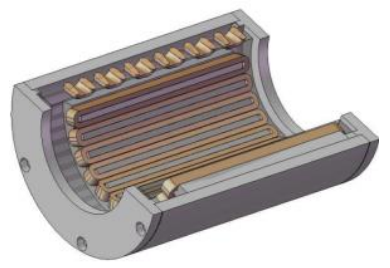

a

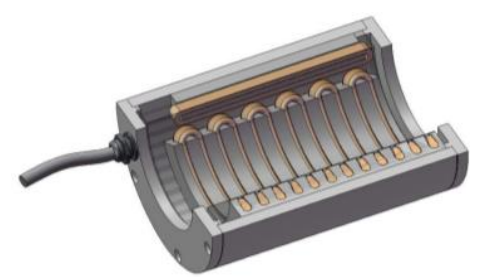

$\mathrm{b}$

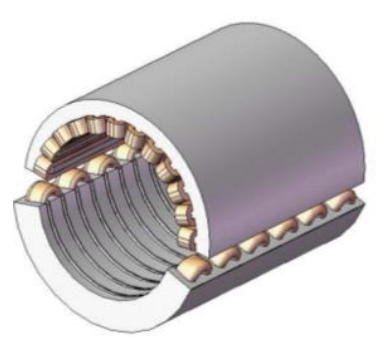

c 


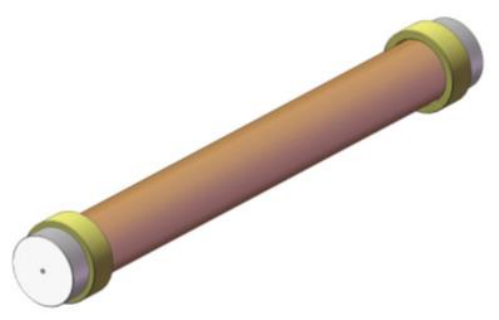

d

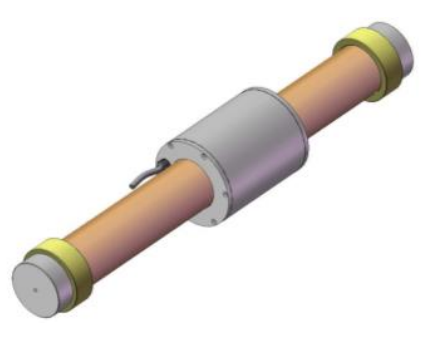

$\mathrm{e}$

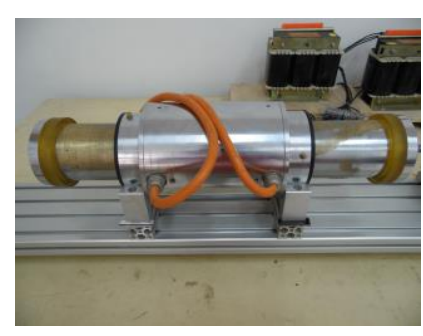

f

Fig. 2. Structure of 2DoFDDIM

a Rotary motion arc-shape stator

b Linear motion arc-shape stator.

c Integrated stator

d Rotor

e Assembly of 2DoFDDIM

f Prototype of 2DoFDDIM

This stator consists of two arc-shaped iron cores, namely a rotary and a linear armature. The former is slotted along the axial direction whereas the latter is slotted in the circumferential direction. They have the same electromagnetic parameters and are assembled orthogonally to form a stator. As for the common solid rotor shared by both stators, a double-layer structure is applied to enhance the performance of the 2DoFDDIM. It is composed of solid steel coated with a thin copper layer. Because the permeability of back steel is far higher than copper, the radial magnetic field will be increased, which will improve the induced magnetic field in the rotor. So the output and power factor of the 2DoFDDIM will be raised especially 
when the slip is small. Although it complicates the mechanical production process, a double layer rotor can provide a better choice. Compared with a single layer rotor, the fluctuation of speed decreases when the double layer rotor is applied to the 2DoFDDIM [11].

When the rotary stator is energized, a rotating magnetic field will be generated. According to the electromagnetic induction principle, the voltage and current will be induced on the rotor surface to produce an electromagnetic torque. In a similar manner, a traveling wave magnetic field will be generated to create a force when the linear stator is powered. If either the rotary or linear stator is energized, the motor produces a linear mechanical motion (single degree). When both are energized, the motor produces a helical motion, namely two-degree mechanical motion [12]-[13]. The main design parameters are listed in Table 1.

Table 1 Main design parameters of the 2DoFDDIM

\begin{tabular}{ccc}
\hline \multirow{2}{*}{ Item } & \multicolumn{2}{c}{ Value/Dimension } \\
\cline { 2 - 3 } & Rotary Part & Linear Part \\
\hline Rated power (PN) & $1.1 \mathrm{~kW}$ & $1.1 \mathrm{~kW}$ \\
Rated voltage (VN) & $220 \mathrm{~V}(\mathrm{Y})$ & $220 \mathrm{~V}(\mathrm{Y})$ \\
Frequency (f) & $50 \mathrm{~Hz}$ & $10 \mathrm{~Hz}$ \\
Pole pairs (p) & 2 & 2 \\
Stator inner diameter & $98 \mathrm{~mm}$ & $98 \mathrm{~mm}$ \\
Stator outer diameter & $155 \mathrm{~mm}$ & $155 \mathrm{~mm}$ \\
Stator axial length & $130 \mathrm{~mm}$ & $156 \mathrm{~mm}$ \\
Air-gap length & $1 \mathrm{~mm}$ & $1 \mathrm{~mm}$ \\
Slot number & 12 & 12 \\
\hline
\end{tabular}

Regarding the structure of the double-layer solid rotor motor, the rotor material along the radial direction is different. Therefore, the electromagnetic field and equivalent circuit parameters cannot be determined accurately by using the equivalent magnetic circuit method or equivalent magnetic impedance method alone. Neither method can derive the distribution of electromagnetic field in the solid steel. Moreover, they are difficult to be used in order to analyse double layer rotor motors since many empirical coefficients are involved. Hence, finite element methods (FEMs) are generally used [7], [14], [15]. However, these require lengthy computational time. Therefore, a multilayer theory is developed to analyse the solid unslotted rotor induction machines [16]. A multilayer model based on the 2D polar coordinates is established [17] while a 
semi-analytical 3-D model based on the Fourier analysis is developed in [18]. The latter is able to calculate the fringing fields in a 3-D slotted cylindrical structure by summing up the linear material properties. Computational time is reduced with an acceptable accuracy. Furthermore, a complex propagation constant can be imported to calculate the rotor parameters [19]- [20].

For 2DoFDDIM, the equivalent magnetic circuit method, equivalent magnetic impedance method and traditional multilayer method have all been used to analyse performance. However, the results are not satisfactory enough. The errors of these three methods are far more than $40 \%$ compared with FEM. In a previous work [14], the permeation depth method (PDM) was applied to analyse its characteristics, while the inner electromagnetic field, as the fundamental theoretical analyses, which is helpful in the future design and optimization, cannot be derived. Apart from this, PDM is not a good way to analyse motor designs with double-layer rotor structures. Therefore, based on the previous research and special structure of 2DoFDDIM, this paper develops a composite multilayer method (CMM) to improve the traditional multilayer theory with a propagation constant method. The CMM, compared with the traditional multilayer method, is more suitable to the 2DoFDDIM with double-layer rotor. The complete CMM program diagram and parameters determination are provided. The relevant calculation of copper layer is also carried out in the computer program, thus there is no need to do any extra work to perform it. However, there are rather intricate electromagnetic field related phenomena such as the coupling effect when both rotary motion and linear motion are considered. Therefore, this paper mainly analyses it from the perspective of a single DOF motor. The distribution of electromagnetic fields for the rotary part is derived. The characteristics of the rotary part and linear part applying the CMM are compared with FEM and experimental results of the 2DoFDDIM.

\section{Analysis and Computation}

\subsection{Multilayer Model of 2DoFDDIM}

In order to derive the CMM model for the 2DoFDDIM, the following assumptions are used:

i) All regions are extended infinitely in a circumferential direction and stretch to infinity in the axial 
direction.

ii) There is no displacement current and magnetic saturation is neglected.

iii) The curvature of the rotor is neglected and the rotor material is isotropic.

iv) The stator windings current is represented by a current sheet at the airgap-stator interface, which is of unlimited length along a circumferential direction and unlimited thickness along a radial direction.

v) The stator slotting effect is included by introducing Carter's coefficient $K_{c}$.

vi) The end effect caused by a tangential current in the solid rotor, is taken into account with the solid rotor end effect coefficient $K_{e}$, which is relevant to stator parameters.

Where $K_{c}=\frac{t_{1}}{t_{1}-v_{1} \delta}$ and $K_{e}=1+\frac{2 \tau}{\pi L_{e}}$. For the 2DoFDDIM, $v_{1}=\frac{\left(b_{01} / \delta\right)^{2}}{4.4+0.75\left(b_{01} / \delta\right)}$, the slot width $b_{01}=2.5 \mathrm{~mm}$, the air-gap length $\delta=1 \mathrm{~mm}$, the tooth pitch $t_{1}=5.6 \mathrm{~mm}$, the polar pitch $\tau=45.16 \mathrm{~mm}$, the stator axial diameter $L_{e}=130 \mathrm{~mm}$. Thus, through derivation $K_{c}=1.21, K_{e}=1.22$.

The CMM model of 2DoFDDIM is established based on the traditional multilayer model [21], in which the rotor is divided into a number of laminar regions, as shown in Fig. 3. Where the first layer is a halfspace, the numbers $1 \sim N-2$ are layers of solid steel and the layers $\mathrm{N}$ and $\mathrm{N}-1$ correspond to the copper layer and air-gap, respectively. The greater the number of secondary layers, the higher the precision and computational time. $\sigma_{i}$ is the conductivity of each layer, $\omega_{i}$ is the angular frequency, $\mu_{i}$ is the permeability of each layer's external surface $(\mathrm{i}=1,2, \ldots, N-2)$. The $\mathrm{x}$-axis is the circumferential direction, the $\mathrm{y}$-axis is the radial direction and the $\mathrm{z}$-axis is axial direction [22].

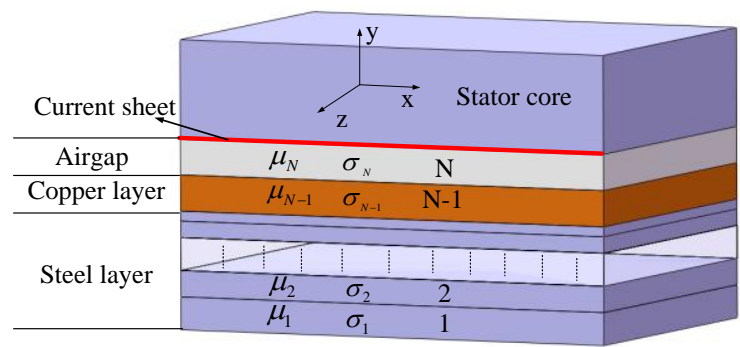

Fig. 3. Multilayer model of the 2DoFDDIM 
The infinitely thin current sheet $J$ is sinusoidal and moves with synchronous speed along the $\mathrm{X}$-axis.

$$
J=J_{0} e^{j\left(\omega_{1} t-a x\right)}
$$

where $J_{0}$ is the magnitude of stator current density, as shown in (2), $\alpha=\pi / \tau, \tau$ is the pole pitch, and $\omega_{1}$ is the angular frequency. $J_{0}$ can be written as:

$$
J_{0}=\frac{2 \sqrt{2} m_{1} K_{d p} W}{\pi D_{i 1}} I_{1}
$$

where $m_{l}$ is the number of phases, $K_{d q} W$ is the series number of armature winding per phase, $I_{l}$ is the stator phase current, and $D_{i l}$ is the inner diameter of the stator.

According to the previous assumptions, it can be supposed as follows:

$$
\left\{\begin{array}{l}
\dot{B}=B_{x} \dot{e}_{x}+B_{y} \dot{e}_{y} \\
\dot{E}=E_{z} \dot{e}_{z}
\end{array}\right.
$$

The equation for the electromagnetic field in a layer can be derived from two of the four Maxwell equations (4):

$$
\left\{\begin{array}{l}
\nabla \times \dot{H}=\dot{J} \\
\nabla \times \dot{E}=-\partial \dot{B} / \partial t
\end{array}\right.
$$

The constitutive relation (5) is the following:

$$
\left\{\begin{array}{l}
\dot{J}=\sigma \dot{E} \\
B_{x}=\mu_{i} H_{x}
\end{array}\right.
$$

Bearing in mind the boundary condition (6) of each layer, the transfer matrix of electromagnetic field, as shown in (7), is conducted by combining (4) and (5) [25].

$$
\left\{\begin{array}{l}
y=0, \quad B_{y}=B_{y i-1}, \quad H_{x}=H_{x i-1} \\
y=b_{i}, \quad B_{y}=B_{y i}, \quad H_{x}=H_{x i}
\end{array}\right.
$$

Where $\dot{B}$ is the magnetic flux density, $\dot{H}$ is the magnetic field intensity, $\dot{E}$ is the electric field intensity, $\dot{e}_{x}, \dot{e}_{y}, \dot{e}_{z}$ are unit vectors of $x, y, z$, respectively. $y=0$ represents the junction of layers $i-1$ and $i, b_{i}$ is the 
layer thickness, $\quad H_{x i}$ and $B_{y i}$ are the tangential magnetic field intensity and radial magnetic flux density of the external surface (i.e. nearer to the air gap) of layer $i$, respectively.

$$
\left[\begin{array}{l}
B_{y i} \\
H_{x i}
\end{array}\right]=\left[\begin{array}{cc}
\cosh \left(\gamma_{i} b_{i}\right) & \frac{1}{\beta_{i}} \sinh \left(\gamma_{i} b_{i}\right) \\
\beta_{i} \sinh \left(\gamma_{i} b_{i}\right) & \cosh \left(\gamma_{i} b_{i}\right)
\end{array}\right]\left[\begin{array}{c}
B_{y i-1} \\
H_{x i-1}
\end{array}\right]
$$

Where $\gamma_{i}=\left(a^{2}+j \omega_{i} \mu_{i} \sigma_{i}\right)^{1 / 2}$ which is related to skin depth, and $\beta_{i}=\gamma_{i} /\left(j a \mu_{i}\right)$. The overall boundary conditions are given as:

$$
\left\{\begin{array}{l}
H_{x 1}=\beta_{1} B_{y 1} \\
H_{x n}=J_{0}
\end{array}\right.
$$

It should be noted that the computational method for the outermost layer (copper layer) is different from the traditional multilayer theory. Moreover, the difficulty in determining parameters involved in the multilayer theory will be overcome in the following sections.

\subsection{Algorithm of the $C M M$}

The parameters of the magnetic field and the machine's equivalent circuit are determined by a CMM iteration program which takes the nonlinear permeability of the steel layer and particularity of the copper layer into consideration. A complete 2DoFDDIM CMM computational program diagram is provided, as shown in Fig. 4, and explained as follows. 


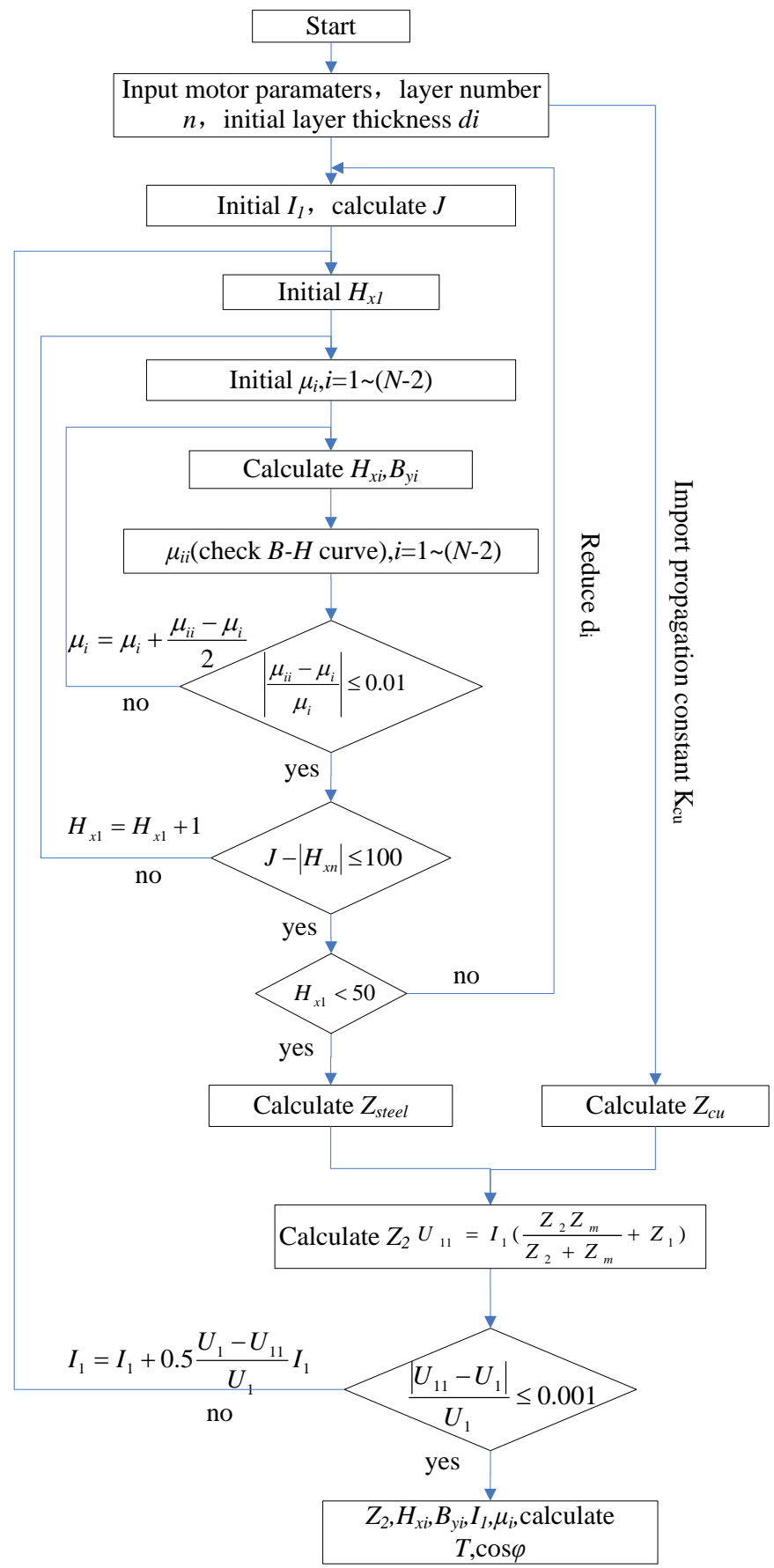

Fig. 4. A complete 2DoFDDIM CMM computational program diagram

In the first cycle, an arbitrary value is assigned to the stator current $I_{1}$ and $\mu_{1} \sim \mu_{N-2}$ to initiate the program. It can be found that the value of $H_{x l}$ increases inversely with the slip. Moreover, in order to fulfil (8) and further improve calculation accuracy, it is better to maintain $H_{x l}$ below 50 when the slip is 1 . It can be achieved by modifying the thickness of each layer for repetitive iterations. According to the transfer matrix 
(7) and boundary condition (8), the magnetic field components $H_{x i}, H_{y i}$, and $B_{y i}(\mathrm{i}=1 \sim N)$ are then determined. Thus, the resultant $H_{i}=\sqrt{H_{x i}^{2}+H_{y i}^{2}}$ is derived. It should be noted here that a loop is created to take the nonlinear $B-H$ characteristics of the lamination material into account. In order to realize it in the iterative program, the $B-H$ curve is represented by a higher order parabola [24].

$$
\mu=K_{t} H^{\frac{1-t}{t}}
$$

For the rotor steel used in 2DoFDDIM, $K_{t} \approx 0.8, t \approx 7$. Applying $H_{i}$ into (9), the new permeability $\mu_{i i}$ is derived. $\mu_{i i}$ is then used for the second cycle. Another loop should be used to ensure $H_{x n}=J_{0}$ of the boundary condition (8). Once the execution is out of the loops, the values of $H_{x i}$ and $B_{y i}$ obtained in the previous program will be used to calculate the steel impedance $Z_{\text {steel }}$, as shown in (10).

$$
Z_{\text {steel }}=\frac{4 m_{1}\left(K_{d p} W\right)^{2}}{\pi D_{2}} Z_{N-2} L_{e} K_{e}
$$

where $D_{2}$ is the diameter of the rotor, $L_{e}$ is the effective length of the iron core, $Z_{N-2}$ is the wave impedance, $Z_{N-2}=\frac{s \omega_{1}}{a} \frac{B_{y N-2}}{H_{x N-2}}$, and $s$ is the slip. $B_{y N-2}$ and $H_{x N-2}$ are the radial magnetic flux density and tangential magnetic field intensity of the steel surface, respectively.

After several attempts, it was found that the iteration speed was slow. The results do not converge if we only apply the multilayer theory to the copper layer of the 2DoFDDIM. To circumvent this difficulty, the propagation constant $K_{c u}$ is imported to calculate the equivalent circuit parameters of copper layer [17], as shown in (11) -(12).

$$
\begin{gathered}
Z_{c u}=\frac{j \omega_{c u} \mu_{c u}}{K_{c u}} \frac{1}{K_{c u} d_{c u}} \frac{L_{e}}{\tau} \frac{K_{e}}{s} \\
K_{c u}=\left(\alpha_{c u}^{2}+\beta^{2}\right)^{1 / 2}
\end{gathered}
$$

where $d_{c u}$ is the thickness of copper layer, $\omega_{c u}$ is the angular frequency of the copper layer, and $\mu_{c u}$ is the copper permeability. The coefficient $\alpha_{c u}$ takes into account the complex propagation of the magnetic field, 
$\alpha_{c u}=\left(j \omega_{c u} \mu_{c u} \sigma_{c u}\right)^{1 / 2}$, where $\sigma_{c u}$ is the copper conductivity. The real constant is $\beta=\pi / \tau$. Thus, the rotor impedance is given by

$$
Z_{2}=\frac{Z_{c u} Z_{\text {steel }}}{Z_{c u}+Z_{\text {steel }}}
$$

Having found $Z_{2}$, it is easier to calculate the stator voltage $U_{11}$. Together with the known parameters of 2DoFDDIM, the new $I_{1}$ and $J_{0}$ can be derived for the next cycle. Once the iterative precision is satisfied, the iteration stops. After numerous loop iterations, accurate results of $I_{1}, \mu_{i}, H_{x i}, B_{y i}$ are then derived. Therefore, the magnetic field distribution in the rotor can be obtained.

The progress of key parameters, such as $U_{11}, I_{1}, H_{x}(n)$, solved in each iteration of the CMM algorithm in Fig. 4 is shown in Fig.5 (slip=0.8). It can be seen that only 12 iterations are needed for $U_{11}$ to converge to $U_{1}, 14$ iterations for $I_{1}$ to reach the steady value and less than 95 iterations for $H_{x}(n)$ to $J_{0}$, in which the elapsed time is only 0.84 seconds for the calculation of the whole program.

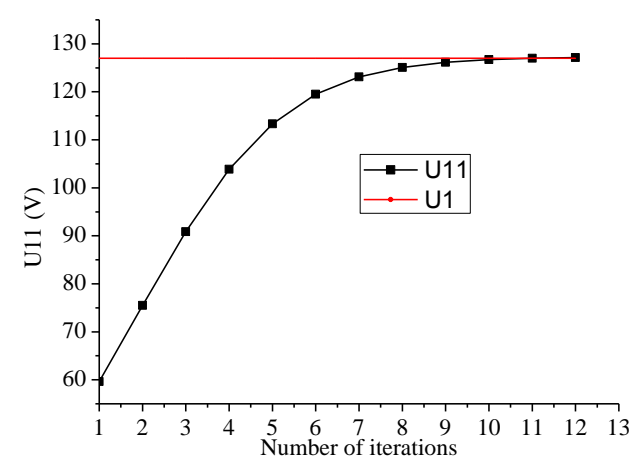

a

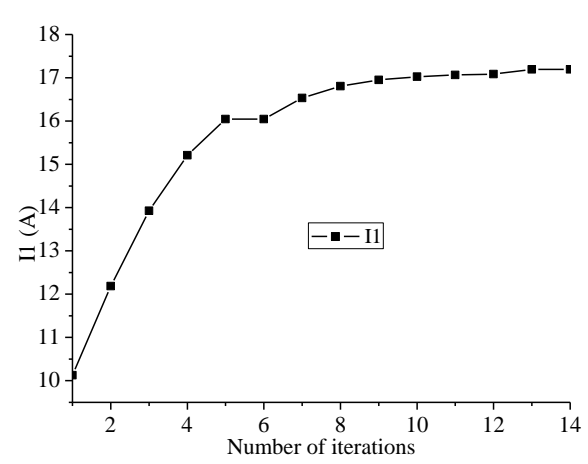

b 


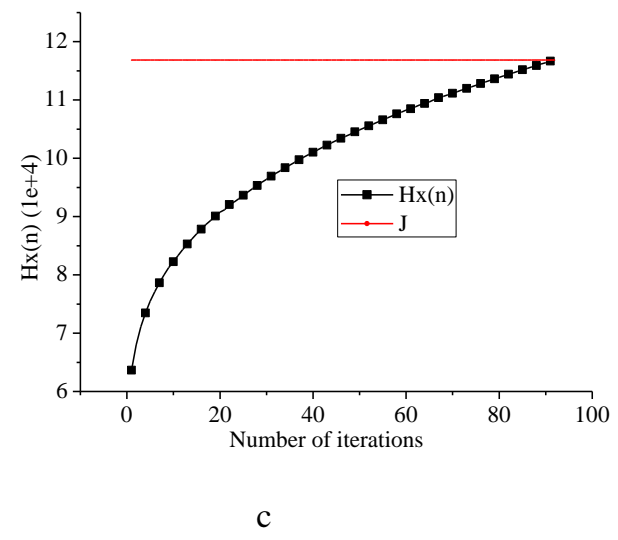

Fig. 5. The progress of key parameters, such as $U_{11}, I_{1}, H_{x}(n)$

a Iteration of $U_{1}$

b Iteration of $I_{1}$.

c Iteration of $H x(n)$

\subsection{Equivalent Circuit}

The per-phase equivalent circuit of the 2DoFDDIM is illustrated in Fig. 6. $R_{1}, R_{m}$, and $X_{1}, X_{m}$ are the resistance and reactance of the primary winding and the excitation, respectively, $R_{c u}, R_{\text {steel, }}$ and $X_{c u}, X_{\text {steel }}$ are the equivalent resistance and reactance of the copper layer and the steel layer, respectively. $R_{2}$ and $X_{2}$ are the rotor equivalent resistance and reactance, which are slip-dependent parameters. $R_{l}, R_{m}, R_{c u}, R_{\text {steel }}, R_{2}$ and $X_{1}, X_{m}, X_{c u}, X_{\text {steel }}, X_{2}$ are the real and imaginary components of the corresponding impedances $Z_{1}, Z_{m}, Z_{c u}$, $R_{\text {steel }}$ and $Z_{2}$, respectively.

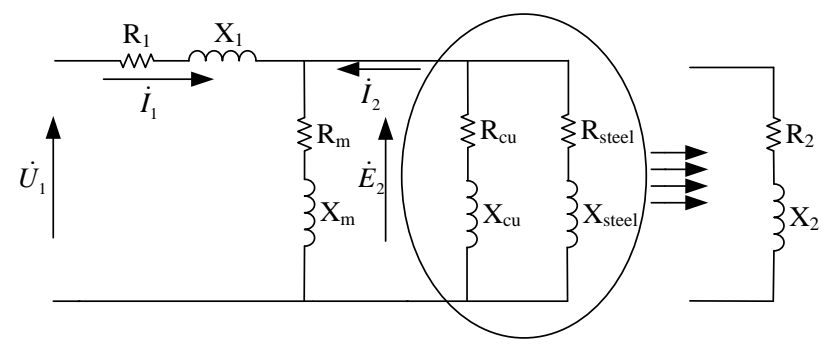

Fig. 6. Equivalent circuit

The equivalent circuit parameters are listed in Table 2. Therefore, the torque, power factor and stator current can be derived accordingly. 
Table 2 Equivalent circuit parameters (slip =1)

\begin{tabular}{cccc}
\hline Item & Value $(\Omega)$ & Item & Value $(\Omega)$ \\
\hline$R_{1}$ & 4.49 & $X_{1}$ & 2.34 \\
$R_{m}$ & 0 & $X_{m}$ & 5.55 \\
$R_{\text {cu }}$ & 2.88 & $X_{\text {cu }}$ & 0.62 \\
$R_{\text {steel }}$ & 15.38 & $X_{\text {steel }}$ & 13.33 \\
$R_{2}$ & 2.51 & $X_{2}$ & 0.70 \\
\hline
\end{tabular}

\subsection{Computation Results}

From the previous analysis, the electromagnetic field parameters $H_{x i}$ and $B_{y i}$ along the radial variation of the rotor material can be obtained, as shown in Fig. 7. Where $H_{x}$ and $B_{y}$ are indicated by the ratio values $H_{x i} / H_{x n-2}$ and $B_{y i} / B_{y n-2}$ respectively. $h_{1}$ represents the radial distance to the external surface of steel.

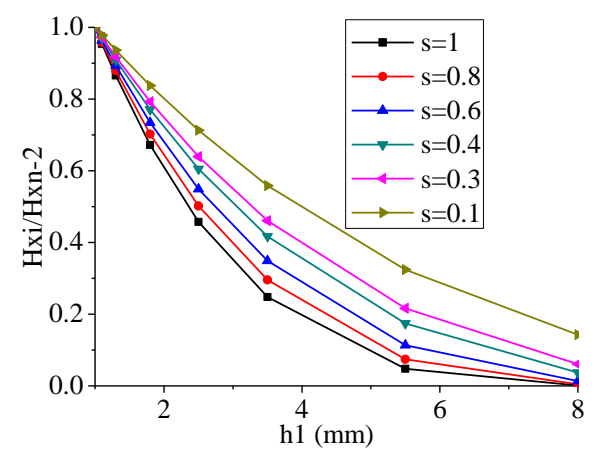

a

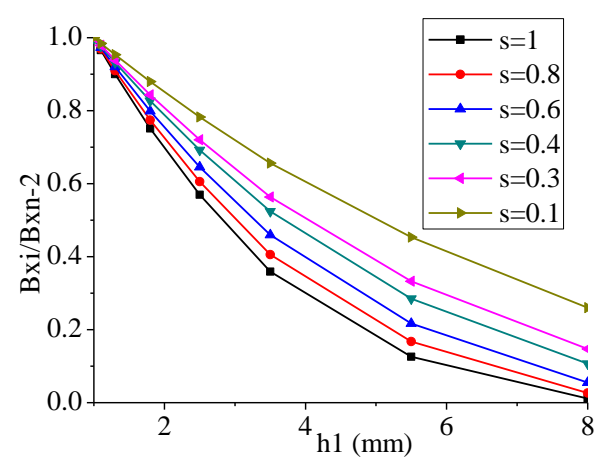

b

Fig. 7. Distribution of electromagnetic field of 2DoFDDIM.

a The tangential magnetic field intensity

$\mathrm{b}$ The radial magnetic flux density

It can be seen that $H_{x}$ and $B_{y}$ decrease along the rotor's radial direction and the attenuation increases when the slip goes up, based on the CMM, which takes the skin effect into account as illustrated before. 


\section{Simulation and Experimental Results}

To verify the CMM, the results of the rotary part of 2DoFDDIM are compared with the results from FEM and prototype tests. A 2D finite element model of the rotary part is established, as shown in Fig. 8 (a). Its flux distribution is shown in Fig. 8 (b) and (c) for a slip of 0.8 .

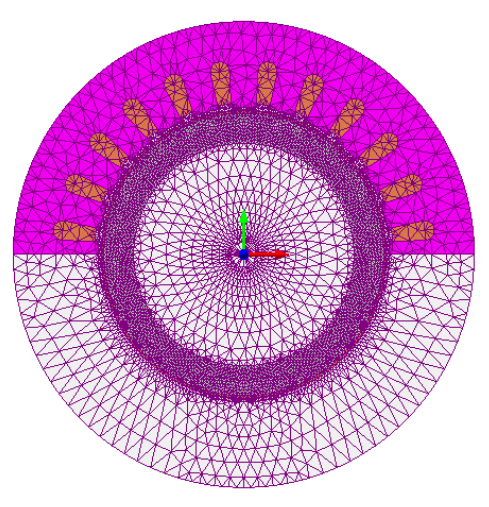

a

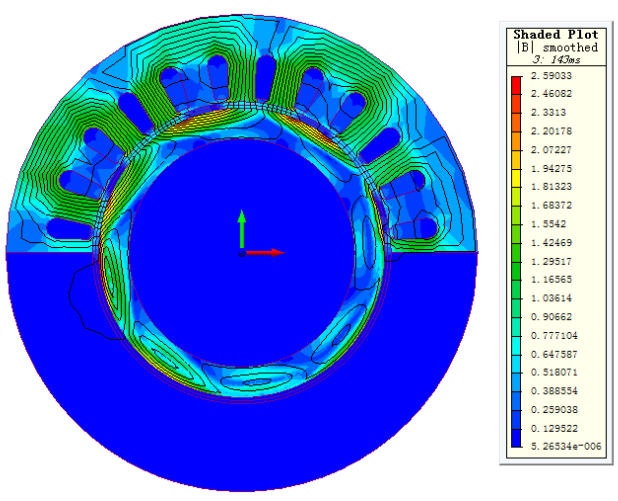

$\mathrm{b}$

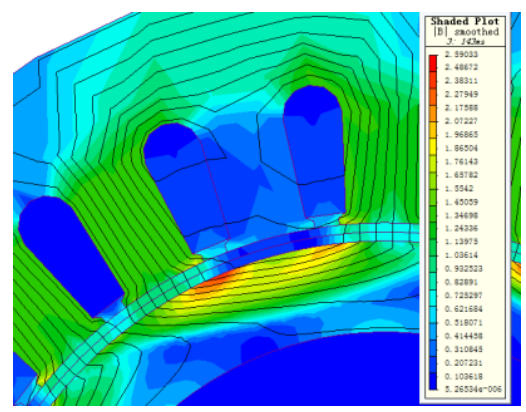

c

Fig. 8. 2D finite element model of the rotary part of the 2DoFDDIM.

a $2 \mathrm{D}$ finite mesh model

b Distribution of flux density

c Part distribution of flux density 
From Fig. 8, the flux density indeed attenuates gradually with growing distance from the external surface of steel. This trend coincides with the results of CMM as well.

The steady-state characteristics of the 2DoFDDIM, in terms of torque, power factor and stator current versus slip, are calculated using CMM and FEM. An overview of the comparison is shown in in Figs. 9-11, Table 3 and Table 4.

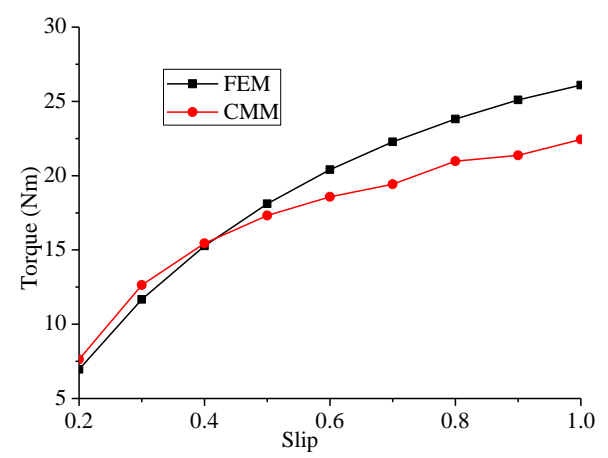

Fig. 9. Torque vs. slip.

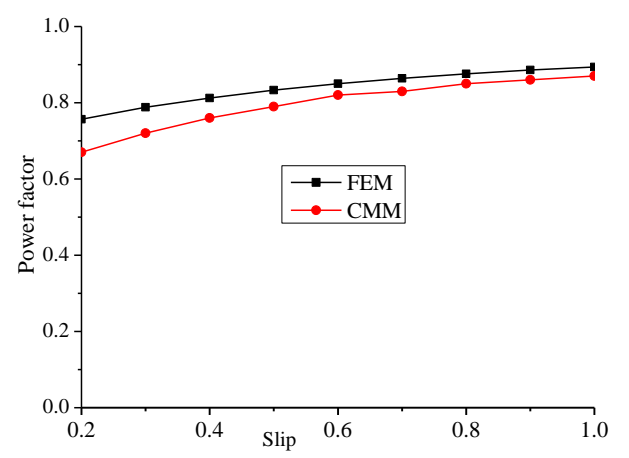

Fig. 10. Power factor vs. slip.

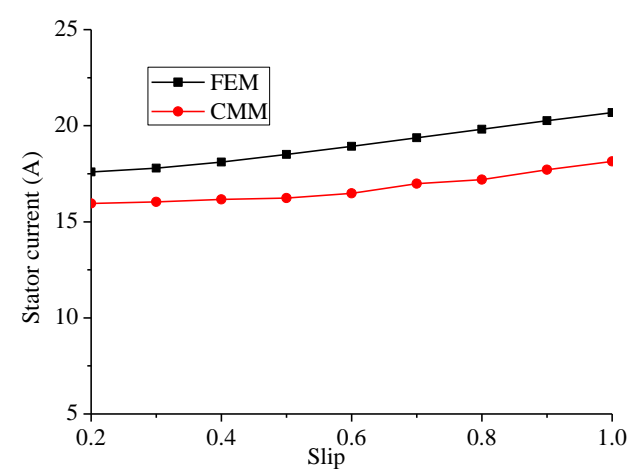

Fig. 11. Stator current vs. slip. 
Table 3 Torque and power factor vs. slip

\begin{tabular}{ccccccc}
\hline \multirow{2}{*}{ Slip } & \multicolumn{3}{c}{ Torque } & \multicolumn{3}{c}{ Power factor } \\
& FEM & CMM & Error & FEM & CMM & Error \\
\hline 0.2 & 6.96 & 7.63 & $9.63 \%$ & 0.75 & 0.67 & $10.67 \%$ \\
0.3 & 11.67 & 12.64 & $8.31 \%$ & 0.79 & 0.72 & $8.86 \%$ \\
0.4 & 15.26 & 15.45 & $1.24 \%$ & 0.82 & 0.76 & $7.32 \%$ \\
0.5 & 18.11 & 17.32 & $4.36 \%$ & 0.84 & 0.79 & $5.95 \%$ \\
0.6 & 20.41 & 18.58 & $8.97 \%$ & 0.85 & 0.82 & $3.53 \%$ \\
0.7 & 22.27 & 19.43 & $12.75 \%$ & 0.86 & 0.83 & $3.49 \%$ \\
0.8 & 23.81 & 20.97 & $11.92 \%$ & 0.876 & 0.85 & $2.97 \%$ \\
0.9 & 25.10 & 21.37 & $14.86 \%$ & 0.885 & 0.86 & $2.82 \%$ \\
1 & 26.09 & 22.43 & $14.03 \%$ & 0.894 & 0.87 & $2.68 \%$ \\
\hline
\end{tabular}

Table 4 Stator current vs. slip

\begin{tabular}{cccc}
\hline \multirow{2}{*}{ Slip } & \multicolumn{3}{c}{ Stator current } \\
& FEM & CMM & Error \\
\hline 0.2 & 17.59 & 15.95 & $9.32 \%$ \\
0.3 & 17.79 & 16.03 & $9.89 \%$ \\
0.4 & 18.1 & 16.16 & $10.72 \%$ \\
0.5 & 18.49 & 16.24 & $12.16 \%$ \\
0.6 & 18.92 & 16.48 & $12.89 \%$ \\
0.7 & 19.37 & 16.98 & $12.33 \%$ \\
0.8 & 19.82 & 17.19 & $13.26 \%$ \\
0.9 & 20.25 & 17.71 & $12.54 \%$ \\
1 & 20.68 & 18.14 & $12.28 \%$ \\
\hline
\end{tabular}

In comparison, the values of power factor are in relatively good agreement, in which the one derived by CMM comes out to be slightly below that from FEM. Furthermore, the values of torque calculated by CMM seem to be in good agreement with that from FEM for small slips and the errors are less than $15 \%$ for large slips. However, the stator current, with the error in the range of $9 \%-14 \%$, is underestimated by the CMM. This underestimation can be explained by local saturation effects, which are neglected in the CMM. Moreover, the errors from the CMM are also caused by neglecting the variation of the stator reactance and the harmonic components. Furthermore, there are some correcting coefficients determined by experience in CMM, which are not always accurate. Moreover, the magnetic field along the axial direction is not referred. Thus, this error is inevitable. However, it is worth mentioning that the computational time of the CMM (less than one second) is far less than that of the FEM and the errors between the CMM and the FEM come out to be less than $15 \%$. Also, the CMM is easier to change than the FEM, which is suitable to the 
preliminary design and optimization of the 2DoFDDIM when many different operating points or designs have to be analyzed.

Fig. 12 and Fig.13 show the prototype testing platform. It consists of:

i) A drive source for 2DoFDDIM.

ii) Frequency converters: YASKAWA V1000. There are two independent frequency converters in which the input is $380 \mathrm{~V} \mathrm{ac}$, and the output is made up of two series of 3-phase PWM waves. They are used to supply the rotary and linear stator windings, respectively.

iii) A controller: TRIO MC403 motion controller. It is used to control the rotary, linear and helical motions of the 2DoFDDIM.

iv) A position sensor: SIKO MSK5000-0241. The axial position and speed of linear motion are detected by grating encoding.

v) AN OMRON limit switch. The switch is used to control the distance of linear motion and provide the switching signal in forward and reverse directions.

vi) A SZKT rotary encoder. The rotary encoder is used to detect the rotary speed and direction of the 2DoFDDIM and provide corresponding feedback signals.

vii) An oscilloscope: YOKOGAWA DL7480.

viii) An operation interface: Motion Perfect v3.2.

The experiment is carried out under the condition of constant voltage frequency ratio $(\operatorname{slip}=0.115)$. The rotary part is fed by a $50-\mathrm{Hz} 220-\mathrm{V}$ supply but the linear part is not powered. The stator current is manifested both on the rotary frequency converter and the oscilloscope. Fig. 14 shows the waveforms from the oscilloscope when the frequency is $20 \mathrm{~Hz}$, and the amplitude of stator current is $117.8 \mathrm{mv}$. Because the ratio of current transformer used here is $100 / 1$, the actual value of stator current is $11.7 \mathrm{~A}$. Then the RMS current is 8.33A. Similarly, the stator currents at different frequencies can be obtained, which are compared with those of the CMM, as shown in Fig. 15. 


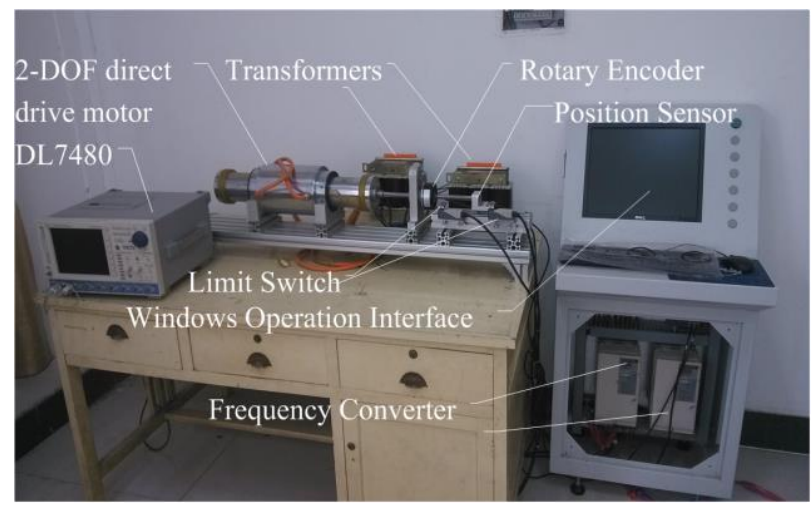

Fig. 12. Photograph of the testing platform system for the 2DoFDDIM
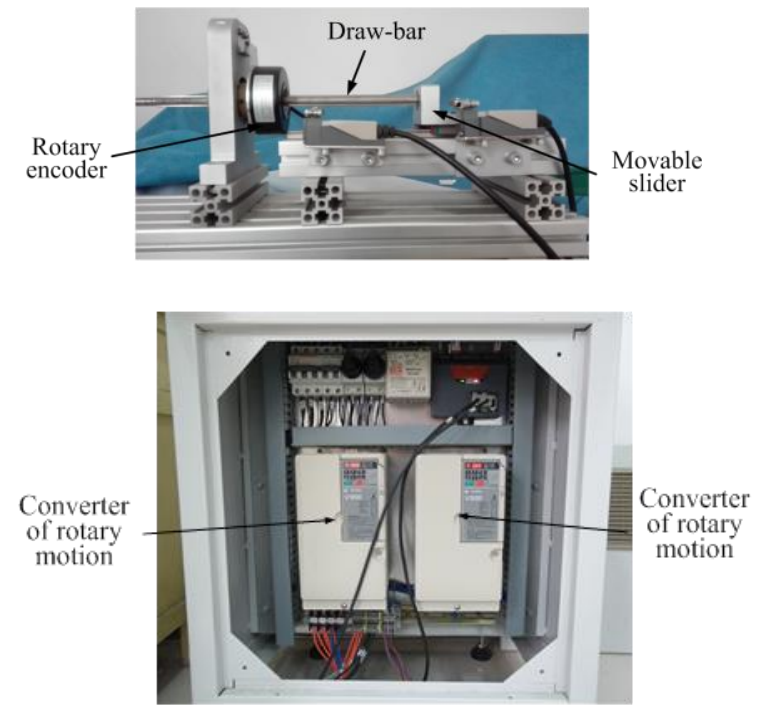

Position sensor Reading head

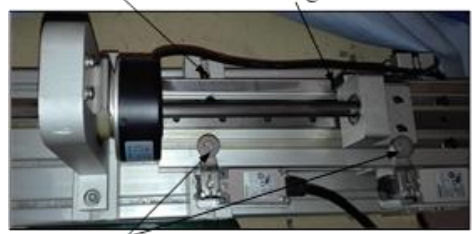

Limit switch

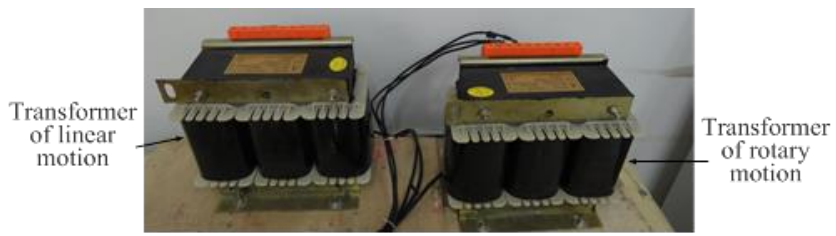

Fig.13. Main parts of the testing platform system for the 2DoFDDIM 


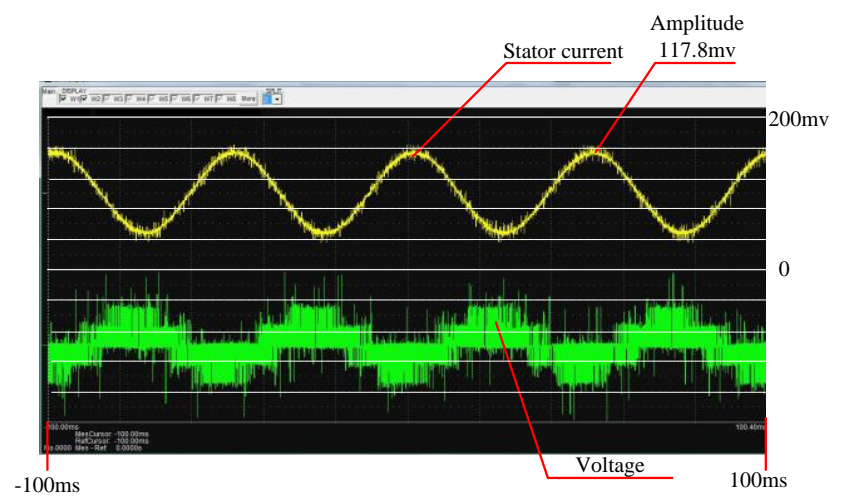

Fig. 14. Current and voltage results from the prototype machine

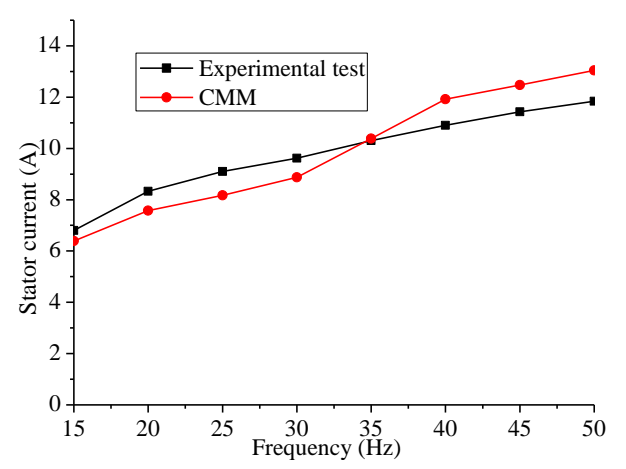

Fig. 15. Stator current (constant V/F control, slip =0.115)

In comparison with the experimental test results, the error from $\mathrm{CMM}$ is less than $10 \%$, which falls within acceptable range.

As for the linear part, a similar analytical method can be used to research its performance. Unfortunately, on account of the restriction of laboratory conditions, such as the limitation of the rotor length, only the current when linear speed is zero is obtained by experimental tests, which is compared with the counterpart values from CMM and FEM, as shown in Table 5. Apart from this, the power factors derived by CMM and FEM are also listed.

Table 5 Comparison of linear results (slip=1, $V / F=180 / 10$ )

\begin{tabular}{ccccc}
\hline Items & CMM & FEM & Experimental test & Error \\
\hline Linear current (A) & 26.52 & 25.59 & 26.73 & $0.79 \%$ (with test) \\
Power factor & 0.54 & 0.49 & $/$ & $10.2 \%$ (with FEM) \\
\hline
\end{tabular}

It can be seen from Table 5, the error of linear current derived by CMM is only $0.79 \%$, much lesser when compared with the values from experimental tests and the power factor calculated by CMM coincides with 
that from FEM, which means that the CMM is also suitable to the linear part of 2DoFDDIM. However, when both the rotary part and linear part are powered and helical motion is happening, the electromagnetic field is much more complicated due to the special coupling effect between two-degree-of-freedom mechanical motions. Therefore, a certain couple effect factor must be imported. The related research is being worked upon and will be involved in the future work.

\section{Conclusion}

An improved electromagnetic analytical method named CMM has been applied to the analysis and performance calculation of the 2DoFDDIM from the perspective of a single DOF motor. A complete computer program is developed to determine key parameters for understanding multilayered rotors in the proposed machine. The propagation constant is imported when dealing with the copper layer. The rotor electromagnetic field distribution is extensively studied using the CMM. The equivalent circuit of the rotary part of the 2DoFDDIM is obtained by calculating the wave impedance. Torque, power factor and stator current of the rotary part under different slip values and of the linear part at zero speed are calculated from the CMM and FEM for comparison purposes. The stator voltage and current are also measured experimentally to verify the CMM. Compared with the FEM, the proposed CMM is simpler and quicker for analysis and performance calculations of the 2DoFDDIM.

\section{Acknowledgements}

This works is supported by National Natural Science Foundation of China under grant 51277054, Project of Key Scientific and Technological Research in Henan Province 152102210101, Henan Polytechnic University Innovation Team T2015-2.

\section{References}

[1] Boglietti, A., Cavagnino, A., Feraris, L., Lazzari, M.: 'Energy-efficient motors', IEEE Industrial Electronics Magazine, Dec 2008, 2, (4), pp. 32-37

[2] Yasukazu, S.: 'Development of 2-degree-of-freedom rotational/linear switched reluctance motor', IEEE 
Trans. Magnetics., Jun 2007, 43, (6), pp. 2564-2566

[3] Yadmellat, P., Shafer, A.S., Kermani, M.R.: 'Design and development of a single-motor, two-dof, safe manipulator', IEEE/ASME Trans. on Mechatronics, Aug 2014, 19, (4)

[4] Cheng, C.-H., Hung, S.-K.: 'A piezoelectric 2-degree-of-freedom nano-stepping motor with parallel design', (Accepted for publication), IEEE/ASME Trans. on Mechatronics, to be published

[5] Pan, J.F., Zou Y., Cheung, N.C.: 'Performance analysis and decoupling control of an integrated rotary-linear machine with coupled magnetic paths', IEEE Trans. on Magnetics, Apr 2014, 50, (2)

[6] Amiri, E.: 'Circuit modeling of double-armature rotary induction motor'. Proc. 39th Annual Conference of the IEEE Industrial Electronics, Dallas, USA, Oct 2014

[7] Markovic, M., Perriard, Y.: 'An analytical solution for the torque and power of a solid-rotor induction motor’. Proc. IEEE International Electric Machines \& Drives Conference, May 2011, pp. 1053-1057

[8] Tsukano, M., Sakaidani, Y., Hirata, K., Niguchi, N., Maeda, S., Zaini, A.: 'Analysis of 2-degree of freedom outer rotor spherical actuator employing 3-D finite element method', IEEE Trans. on Magnetics, May 2013, 49, (5), pp. 2233-2236

[9] Meessen, K.J., Paulides, J.J.H., Lomonova, E.A.: 'Analysis of a novel magnetization pattern for 2-dof rotary-linear actuators', IEEE Trans. on Magnetics., Nov 2012, 48, (11), pp. 3867-3870

[10]Mendrela, E.A., Gierczak, E.: ‘Double-winding rotary-linear induction motor', IEEE Trans. on Energy Conversion, Mar 1987,7, (3), pp.32-32

[11]Fleszar, J., Mendrela, E.A.: 'Twin-armature rotary-linear induction motor', IEE Proceedings B Electric Power Applications, May 1983, 130, (3), pp. 186 - 192

[12]Si, J., Si, M., Xu, X., Feng, H., Wang, X.: 'Effects of rotor conduction material and air gap length on performance of linear arc-shaped induction motor', Electric Machines and Control, 2012, 16, (10), pp. $31-37$

[13]Si, J., Si, M., Feng, H., Xu, X.: 'Research status and development of two-degree-of-freedom direct 
drive motor', Transactions of China Electrotechnical Society, 2013, 02, pp. 97-107

[14]Si, J., Feng, H., Ai, L., Hu, Y., Cao, W.: 'Design and analysis of a 2-dof split-stator induction motor', IEEE Trans. on Energy Conversion., Apr 2015, 30, (3), pp.1200-1208

[15]Lee, J.-S., Kim, D.-K., Baek, S.-W., Rhyn, S.-H., Kwon, B.-I.: 'Newly structured double excited two-degree-of-freedom motor for security camera', IEEE Trans. on Magnetics., Nov 2008, 44, (3), pp. 4041-4044

[16]Lim, D.-K., Yi, K.-P., Woo, D.-K., Yeo, H.-K., Ro, J.-S., Lee, C.-G., Jung, H.-K.: 'Analysis and design of a multi-layered and multi-segmented interior permanent magnet motor by using an analytic method', IEEE Trans. on Magnetics., Jan 2014, 50, (6), article seq no. 8201308

[17]Tang, X., Liu, S.: 'Analysis of solid unslotted rotor induction machines using multi-layer theory', Transactions of China Electrotechnical Society, 1989, 2, pp. 29-34

[18]Guo, S., Zhou, L., Yang, T.: 'An analytical method for determining circuit parameter of a solid rotor induction motor'. Proc. 15th International Conference on Electrical Machines and Systems, 2012, pp.1-6

[19]Meessen, K.J., Gysen, B.L.J, Paulides, J.J.H., Lomonova, E.A.: 'General formulation of fringing fields in 3-D cylindrical structures using Fourier analysis', IEEE Trans. on Magnetics., Apr 2012, 48, (18), pp. $2307-2323$

[20]Toliyat, H.A., Kliman, G.B.: 'Handbook of electric motors 2nd ed.' (Marcel Dekker, 2004), pp. $134-138,273-276$

[21]Gieras, J.F.: 'Performance calculation for a high-speed solid-rotor induction motor', IEEE Trans. on Industrial Electronics, Jun 2012, 59, (6), pp. 2689-2700

[22]Boldea I., Babescu M.: 'Multilayer theory of d.c. linear brakes with solid-iron secondary', Proceedings of the Institution of electrical Engineers, Mar 1976, 2, pp. 220-222

[23]Freeman, E.M.: 'Equivalent circuits from electromagnetic theory: low frequency induction devices', 
Proc. IEE (United Kingdom), Oct 1974, 121, (10), pp.1117-1121

[24]Fu, F., Tang, X.: “'Handbook of asynchronous motor design, $2^{\text {nd }}$ ed.', (China Machine Press, 2007), pp. 603 